\title{
Central and Peripheral Effects of Transcutaneous Acupuncture Treatment for Nausea in Patients with Diabetic Gastroparesis
}

\author{
Irene Sarosiek, ${ }^{1}$ Gengqing Song, ${ }^{1}$ Yan Sun, ${ }^{1}$ Hugo Sandoval, ${ }^{2}$ Stephen Sands, ${ }^{2}$ Jiande Chen, ${ }^{3}$ and Richard W McCallum ${ }^{1 *}$ \\ ${ }^{I}$ Department of Internal Medicine, Texas Tech University Health Sciences Center, Paul L. Foster School of Medicine, El Paso, TX, USA; ${ }^{2}$ Center of \\ Excellence of Neuroscience, Texas Tech University Health Sciences Center, Paul L. Foster School of Medicine, El Paso, TX, USA; and ${ }^{3}$ Division of \\ Gastroenterology and Hepatology, Johns Hopkins Center of Neurogastroenterology, Baltimore, MD, USA
}

\section{Background/Aims}

Nausea, an unpleasant symptom of diabetic gastroparesis (DMGP), has been reported to be alleviated by needleless transcutaneous electrical acupuncture (TEA). Our study was designed to utilize electroencephalography (EEG) and electrogastrography (EGG) recordings to investigate the central and peripheral responses of TEA in the treatment of nausea in DMGP patients.

\section{Methods}

Eleven DMGP subjects underwent simultaneous EEG and EGG testing while grading the severity of nausea following 30-minute intervals of: (1) baseline, (2) visual stimulation (VS) to provoke more nausea, (3) active VS together with TEA, and (4) TEA alone, and a final 15-minute recording without any intervention.

\section{Results}

The nausea score was increased to $5.9 \pm 1.5$ with VS $(P<0.05$, vs $3.5 \pm 1.0$ at baseline), then reduced to $3.5 \pm 1.2$ with VS plus TEA, and to $2.5 \pm 1.3$ with TEA alone, while it continued at a score of $2.9 \pm 1.0$ post TEA (all significant, $P<0.05$, vs VS without TEA). The mean percentage of normal gastric slow waves was decreased to $60.0 \pm 5.7 \%$ with VS $(P<0.05$, vs $66.6 \pm$ $4.5 \%$ at baseline), then improved to $69.2 \pm 4.8 \%$ with VS plus TEA, and maintained at $70 \pm 3.6 \%$ with TEA alone. During initial VS, EEG signals showed right inferior frontal activity as the prominent finding, but during VS with TEA, left inferior frontal activity predominated.

\section{Conclusions}

In DMGP, TEA improves gastric dysrhythmia and ameliorates nausea. TEA treatment of nausea provoked by VS resulted in a change of dominance from right to left inferior frontal lobe activity. These data provide new understandings of peripheral and central mechanisms for nausea, and potential future directions for DMGP treatment approaches.

(J Neurogastroenterol Motil 2017;23:245-253)

Key Words

Acupuncture; Diabetes; Electroencephalogram; Electrogastrogram; Gastroparesis

Received: June 24, 2016 Revised: October 12, 2016 Accepted: October 23, 2016

(a) This is an Open Access article distributed under the terms of the Creative Commons Attribution Non-Commercial License (http://creativecommons. org/licenses/by-nc/4.0) which permits unrestricted non-commercial use, distribution, and reproduction in any medium, provided the original work is properly cited.

${ }^{*}$ Correspondence: Richard W McCallum, MD, FACP, FRACP (AUST) FACG, AGAF

Department of Internal Medicine, Texas Tech University Health Sciences Center, Paul L. Foster School of Medicine, 4800 Albert Avenue, El Paso, TX 79905, USA

Tel: +1-915-215-5218 (direct office: +1-915-545-6634), Fax: +1-913-706-6746, E-mail: richard.mccallum@ttuhsc.edu

Irene Sarosiek and Gengqing Song contributed equally to this work. 


\section{Introduction}

Gastroparesis is an upper gastrointestinal (GI) disorder characterized by delayed gastric emptying without any mechanical obstruction of the gastric outlet or small intestine. ${ }^{1-3}$ The main etiologies are diabetes, idiopathic and post-gastric, and esophageal surgical settings where vagal nerves were damaged. Common symptoms of gastroparesis are early satiety, fullness, bloating, nausea, and vomiting. The management of gastroparesis is challenging due to a limited number of available medications, their adverse effects and variable symptomatic improvement of the patients.

Minimally invasive techniques including acupuncture have frequently been used for the treatment of GI symptoms predominantly in Eastern countries. The most common acupuncture points (acupoints) for alleviating GI symptoms are the Neiguan (PC6) and the Zusanli (ST36). Manual acupuncture at the PC6 acupoint is antiemetic in patients undergoing gynecological surgery. ${ }^{4,5}$ Electroacupuncture at the PC6 acupoint, through providing electrical stimulation, is as effective as manual needling. ${ }^{4}$ Electroacupuncture at the PC6 acupoint reduces chemotherapy induced nausea and vomiting compared to sham acupoints. ${ }^{4,6}$ Recently, we have studied the efficacy of transcutaneous electroacupuncture (TEA) in healthy volunteers $^{7}$ as well as patients with gastroparesis using a sham controlled trial ${ }^{8}$ where electrical stimulation was applied to acupoints via surface electrodes without needles. This method was well tolerated and was efficacious.

As far as we know, no studies have assessed the anatomical function of the CNS in diabetes with nausea. However, several studies have investigated abdominal pain in diabetes, utilizing the electroencephalography (EEG) and evoked potentials (EPs). ${ }^{9,10}$ These studies have provided some insight into the nature of diabetes-related GI complications eg, diabetic neuropathy, suggesting some critical roles for the CNS in symptom generation. Inferior frontal lobe asymmetry association with pleasant and unpleasant feelings has been reported by Craig. ${ }^{11}$ Here we have summarized imaging results and described how right inferior frontal is more active during nausea in contrast to left inferior frontal being more dominant during pleasant stimuli eg, improvement of nausea symptoms.

Electrogastrography (EGG) has been accepted as a method to record impaired myoelectrical activity such as abnormal frequency of the gastric slow wave, low amplitude and/or uncoupling of slow waves. $^{12-17}$

Based on this background overview, our goals in this current pilot study were to investigate the central and peripheral responses to TEA in diabetic gastroparetic patients with predominant symptoms of nausea and vomiting. We have studied, therefore, (1) brain networks through analysis of EPs recorded by EEG, (2) gastric function using EGG recordings, (3) the effects of TEA on controlling nausea, and (4) correlation of findings on EEG and EGG recordings with nausea scores before, during, and after TEA therapy.

\section{Materials and Methods}

\section{Subjects}

Eleven nauseated diabetic gastroparesis (DMGP) patients (11 females, mean age 51 [23-71] years old) with established gastroparesis by scintigraphic documentation were enrolled in this protocol which was approved by the Institutional Review Board of Texas Tech University Health Sciences Center, Paul L. Foster School of Medicine. Written consent was obtained from all subjects.

\section{Patient Recruitment and Screening}

\section{Recruitment}

Patients with DMGP were recruited from Dr McCallum's Clinic and treatment programs at the Texas Tech University Health Science Center at El Paso. During the recruitment process, prequalified patients were presented with a study specific consent form. After being read by the patients, it was also explained by the patient based on the "tell-me-back" method, in order to completely secure the understanding of all tests and other relevant steps of the study.

\section{Screening}

Patients with documented DMGP were asked a standardized series of questions to determine whether they met criteria for participation with respect to age, prior medical history, and having been diagnosed with DMGP as well as having daily nausea present. $\mathrm{Pa}$ tients who qualified as eligible for participation were asked a further set of standardized questions about their ability to comply with the study protocol regarding EEG and EGG recordings, the use of the TEA, and sign a medical record release to allow verification of their medical history and DMGP diagnosis.

\section{Inclusion criteria}

Inclusion criteria include: (1) males and females between ages 18-75 year old diagnosed with DMGP for at least 6 months; (2) symptomatic DMGP with nausea as a daily symptom with or with- 
out occasional vomiting; (3) at least 1 severe gastroparetic symptom or 2 moderate gastroparetic symptoms (see assessment of gastroparetic symptoms); (4) abnormal standard 4 hour scintigraphic gastric emptying results obtained in the past 2 years; (5) upper endoscopy or upper GI within the last year showing no evidence of obstruction or peptic ulcer; (6) no changes in patients clinical presentation which could require endoscopic testing; (7) subjects with high probability for compliance and completion of the study; and (8) average daily glucose level of $<275 \mathrm{mg} / \mathrm{dL}$ and $>75 \mathrm{mg} / \mathrm{dL}$.

\section{Exclusion criteria}

Exclusion criteria include: (1) uncontrolled diabetes with serum glucose greater than $300 \mathrm{mg} / \mathrm{dL}$; (2) previous gastric surgeries eg, surgical resection of the stomach, evidence of small bowel obstruction, and gastric bypass surgery for obesity; (3) severe renal failure with hemodialysis; (4) active treatment for malignancy; (5) patients requiring frequent hospitalization for frequent vomiting; and (6) patients requiring narcotics for pain management.

\section{Experimental Protocol (See Fig. 1)}

Eleven nauseated DMGP patients were fasted for at least 6 hours prior to the study. Patients were permitted to continue their usual medications for severe gastroparesis symptoms, specifically nausea. Prior the study, all EEG, EGG, and TEA electrodes were positioned on skin at the specific topographic points described under each of the tests. Patients underwent concurrent EGG and EEG recordings while also grading the nausea severity on a $0-10$ points scale (see method session below) during the follow sequence of experiment: 30-minute baseline; 30-minute visual stimulation by a flashing/rotating checkerboard a method previously utilized by our research team; ${ }^{18} 30$-minute visual stimulation plus TEA therapy (at PC6 and ST36 acupoints); 30-minute with TEA alone; and 15-minute post TEA. During EEG recordings, subjects were in a partially reclined position, while a visual stimuli consisting of a flashing rotating checkerboard was presented. Assessment of gastroparesis symptoms with special focusing on nausea scores were completed throughout all phases of this project.

\section{Transcutaneous electroacupuncture}

Location of acupuncture points (see Fig. 2). Two acupoints, ST36 (Zusanli) and PC6 (Neiguan) were chosen for the TEA therapy. These two acupoints have been traditionally used to heal upper GI symptoms and frequently chosen in research studies, including our previous studies. ${ }^{7,8,19,20}$ PC6 is a common acupuncture point used for the treatment of nausea and vomiting, whereas ST36 is one of the most frequently used acupuncture points for the treatment of gastric diseases. These 2 points were used together in a previous study in humans and were be effective in treating dyspeptic symptoms and improving gastric motility. ${ }^{11}$ The location of ST36 (Zusanli) is at the depression inferior to tibia tubercle and one finger-breadth (the patient's thumb finger) from the anterior crest
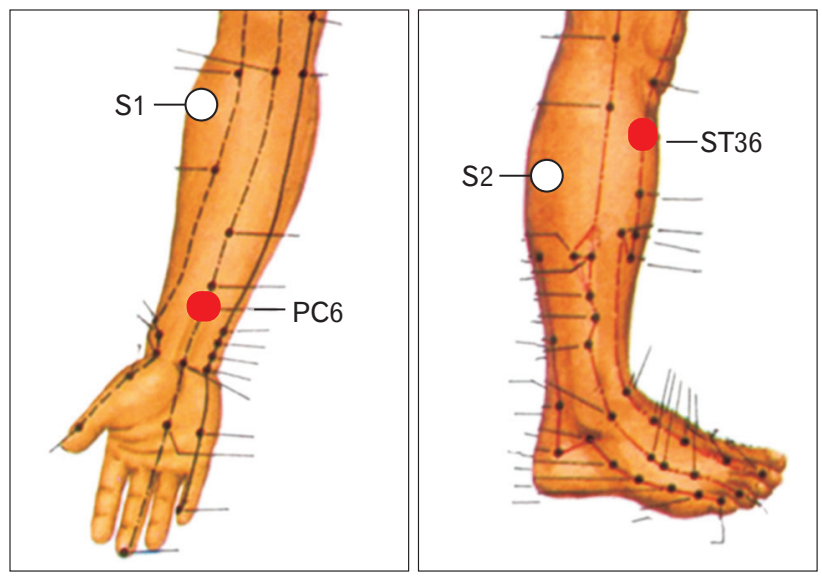

Figure 2. Locations of acupuncture points (PC6 Neiguan and ST36 Ausanli). The lines on the leg and arm are meridians and the dots are various acupuncture points. Points S1 and S2 represent sham location of acupoints.

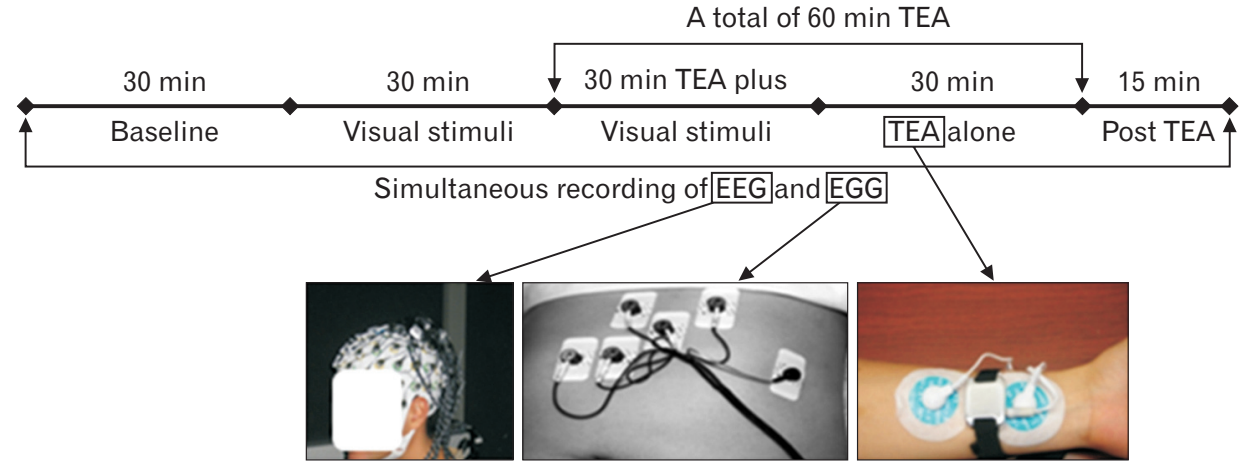

Figure 1. The experimental design of the protocol illustrating the flow of particular phases, including electroencephalography (EEG) plus electrogastrography (EGG) recordings, presentation, and application of transcutaneous electroacupuncture (TEA) therapy. 
of the tibia. Anatomically, it is between the muscle anterior tibialis and muscle extensor digitorumlongus. Superficially, there are the lateral sural cutaneous nerve and the cutaneous branch of the saphenous nerve at this acupoint. Deeply, there is the peroneal nerve.

PC (Pericardial meridian) 6 (Neiguang) is located at the junction of the distal one-sixth and the proximal five-sixth from the transverse crease of the wrist to the transverse cubital crease, between the tendons of palmaris longus and flexor carpi radialis. The medial antebrachial cutaneous nerve, the median nerve (deeper) and the anterior interrosseous nerve (deepest) are distributed at the location.

Transcutaneous electroacupuncture. TEA was performed continuously for 60 minutes immediately after the 30-minute recordings with visual stimuli (Fig. 1). Both PC6 and ST36 were used for delivery of TEA. This method was developed and reported by our group. ${ }^{8}$ For TEA through PC6, 2 cutaneous electrodes were placed prior to baseline period (Fig. 2): one right at the acupoint and the other 4 to $6 \mathrm{~cm}$ away from the acupoint along the meridian of the acupoint. For TEA via ST36 (Fig. 2): one cutaneous electrode right at the acupoint and the other, $4 \mathrm{~cm}$ down from ST36 along the same meridian.

Trains of pulses used for TEA were delivered by wrist watch like device (Fig. 1). Pulses in each train had a frequency of $25 \mathrm{~Hz}$ and an amplitude range of 2-10 $\mathrm{mA}$, based on patient tolerability, specifically tingling or vibratory sensation in the forearm or the leg. These parameters were previously shown to be effective in treating GI symptoms in patients with gastroparesis, scleroderma and functional dyspepsia. ${ }^{7,8,12,19-22}$

\section{Measurements of electrogastrography and electroen- cephalography}

Recording and analysis of the electrogastrography. A noninvasive method similar to electrocardiography, called electrogastrography, was used in this study. ${ }^{13,16,17}$ The EGG was recorded using a multichannel recording device which is standard Food and Drug Administration-approved clinical equipment (Digitrapper EGG; MedtronicSynectics, Shoreview, MN, USA). The device consists of four identical amplifiers with cut-off frequencies of 1.8 cpm and $16.0 \mathrm{cpm}$. The data were converted from analogue to digital with a sampling frequency of $1 \mathrm{~Hz}$ on line and stored on an IBM-compatible computer. Patients remained at 30 degrees head elevation and were required to remain motionless in order to avoid possible artifacts derived from body movements. Fourchannel EGG signals were recorded. Six silver-silver chloride ECG electrodes were placed on the abdomen. Electrode position was as follows: 4 active electrodes (electrode 3, $2 \mathrm{~cm}$ above the mid-point between the xiphoid process and the umbilicus; electrode $4,4 \mathrm{~cm}$ right to electrode 3 ; electrode $2,4 \mathrm{~cm} 45^{\circ}$ upper left to electrode 3 ; and electrode $1,4 \mathrm{~cm} 45^{\circ}$ upper left to electrode 2 ), a reference electrode (at the cross point of a horizontal line on electrode 1 and a vertical line on electrode 3), and a ground on the left costal margin.

Recording and analysis of the slow cortical evoked potential. Slow wave cortical activity was recorded with a DC amplifier (Electrode Arrays, El Paso, TX, USA). Subjects were fitted with an Electrode Arrays 68 channel cap system. ${ }^{23}$ Electrodes were distributed evenly to facilitate accurate source reconstruction model described in literature. ${ }^{24}$ The reference point was located between $\mathrm{CZ}$ and $\mathrm{CPZ}$ electrodes. The ground lead was placed at AFZ. EEG was amplified with an Electrode Arrays EA136 system at 1 $\mathrm{kHz}$ per channel. The EEG filter was provided by DC-200 Hz. Electrodes were sintered $\mathrm{Ag} / \mathrm{AgCl}$ type and they were individually shielded. Impedances for each electrode were set below $5 \mathrm{k} \Omega$. The EEG cap was placed on the subject's head, and gel was applied to every electrode, and the cap was then connected to an EEG amplifier. If necessary, skin was gently abraded with a blunt needle or an acupuncture needle in order to bring down impedances. Subjects sat still during the study. After the completion of the experiment, the cap and EEG electrodes were removed. The subject had the option of washing his/her hair after the study.

The experiment was controlled by Sensorimotoric Systems (SensoMotoric Instruments, Inc, Boston, MA, USA), equipped with Experiment Center software. Stimulus onset was monitored by a digital port connected to the parallel port. In addition, a phototransistor monitored onset and offset on a mirrored subject monitor for simultaneous recordings in order to guarantee precise timing.

An off-line DC drift correction method based on a previous study $^{25}$ was implemented to correct for DC drift artifacts. To accurately localize the source of the EEG activity measured, a technique based on a source reconstruction method was performed. Current density source reconstruction techniques were used and constrained to the cortical surface. A boundary element model using the Montreal Neurological Institute standardized brain in the CURRY source reconstruction software (Compumedics Neuroscan, Inc, Melbourne, Australia) was used as a volume conductor. ${ }^{26}$ The boundary element model calculates the electric potential of a current source by using triangulated brain compartments representing the brain, skull, and skin. ${ }^{27}$ Standardized low-resolution brain electromagnetic tomography source reconstruction (sLORETA) was the method that computes images of electric neuronal activity from EEG $^{28}$ To quantify and perform statistical comparison, regional 
dipoles in the orbital frontal and inferior frontal gyrus structures were computed using the BESA software (BESA Gmbh, Munich, Germany). The area under the curve for each source waveform was computed. The Montreal Neurological Institute brain anatomical MRI is provided by CURRY as a 3 dimensional image for the overlay of functional results. Skin was chosen for threshold segmentation and electrode positions for each study were imported and arranged in accordance with the $10-10$ system. ${ }^{23}$ A pre-stimulus baseline correction interval of -1 second was selected. This interval was also used for noise estimation and signal-to-noise ratio measurements.

\section{Visual analog scale}

A visual analogue scale (VAS) was used to assess the severity of nausea during each phase of the study. ${ }^{29}$ VAS is a measurement instrument that evaluates a characteristic of values which cannot be measured directly. VAS is a horizontal line, $100 \mathrm{~mm}$ in length, described by a specific number, 0 to an extreme 10 , at each end. The patient marks on the line the point representing subjective perception of the current state, which when is read in millimeters from the left end to the point marked by the patient, shows the progression/ regression of severity of nausea, as an expression of changes in individual patients.

\section{Data Analysis}

Data analyses were performed to assess. (1) Findings and distribution of EEG patterns in patients with nausea: the EEG data was analyzed by using independent component analysis and analysis of variance. To accurately localize the source of the EEG activity measured, source reconstruction was performed. Both dipole fit and current density source reconstruction techniques were used. ${ }^{28}$ (2) The percentage of normal slow waves on EGG recordings, and identification of slow wave dysrhythmia in nauseated gastroparesis patients: previously validated spectral analysis methods were used to derive the following parameters from the $\mathrm{EGG}^{13,16,17}$ : (a) dominant frequency and power of the slow waves, (b) the percentage of normal 2-4 cycles/min slow waves, and (c) the percentage of tachygastria, bradygastria, or arrhythmia. (3) Effects of TEA on EEG and EGG patterns, as well as changes of nausea scores: the baseline data were compared with those with TEA using the paired Student's $t$ test. Correlations between EGG, EEG, and symptoms were obtained using Pearson correlation analysis.

\section{Statistical Methods}

All data were presented as means $\pm \mathrm{SE}$. The analysis of variance (ANOVA) was used to compare differences in nausea scores, EGG parameters and EEG activity among 3 or more recording periods. The paired Student's $t$ test was applied to investigate differences in any of the parameters between two sessions. Statistical significance was assigned for $P<0.05$.

\section{Results}

\section{Effects of Visual Stimuli on Severity of Nausea, and Responses to Transcutaneous Electroacupuncture Treatment}

The mean nausea score in 11 gastroparetic patients was $3.5 \pm$ $1.0(\mathrm{SE})$ at the baseline and increased to $5.9 \pm 1.5$ with visual stimuli $(P<0.01)$. The nausea score then decreased to $3.5 \pm 1.2$ with visual stimuli plus TEA, decreased further to $2.5 \pm 1.3$ with TEA alone, and was sustained $2.9 \pm 1.0$ post TEA. All results were significantly reduced $(P<0.05)$ versus the level of nausea with visual stimulation and before TEA was initiated (Fig. 3).

\section{Effects of Visual Stimuli on Gastric Slow Waves and Responses to Transcutaneous Electroacupuncture Treatment}

The mean percentage of normal gastric slow waves was decreased to $60.0 \pm 5.7 \%$ with visual stimuli with no TEA $(P<$

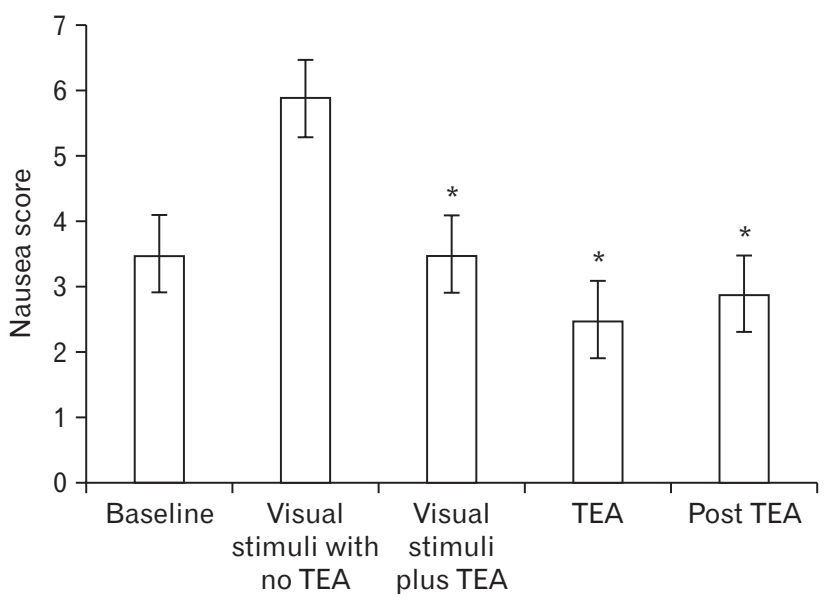

Figure 3. Nausea scores during the different phases of the experimental research protocol. ${ }^{*} P<0.05$ vs the corresponding visual stimuli with no transcutaneous electroacupuncture (TEA). 
0.05 vs $66.6 \pm 4.5 \%$ at baseline); then improved to $69.2 \pm 4.8 \%$ with visual stimuli plus TEA, improved further to $70 \pm 3.6 \%$ with TEA alone and was sustained $70 \pm 4.8 \%$ post TEA (all significant, $P<0.05$, vs visual stimuli with no TEA) (Fig. 4).

\section{Effects of Visual Stimuli on Inferior Frontal Lobe Activity and Responses to Transcutaneous Electroacupuncture Treatment}

During initial visual stimulation, based on construction of the EEG signals the right inferior frontal activity was prominent but when the visual stimuli was presented simultaneously with TEA, left inferior frontal activity became predominant. This effect of TEA on left inferior frontal activity was also observed after TEA had stopped suggesting a carryover effect resulting from this treat-

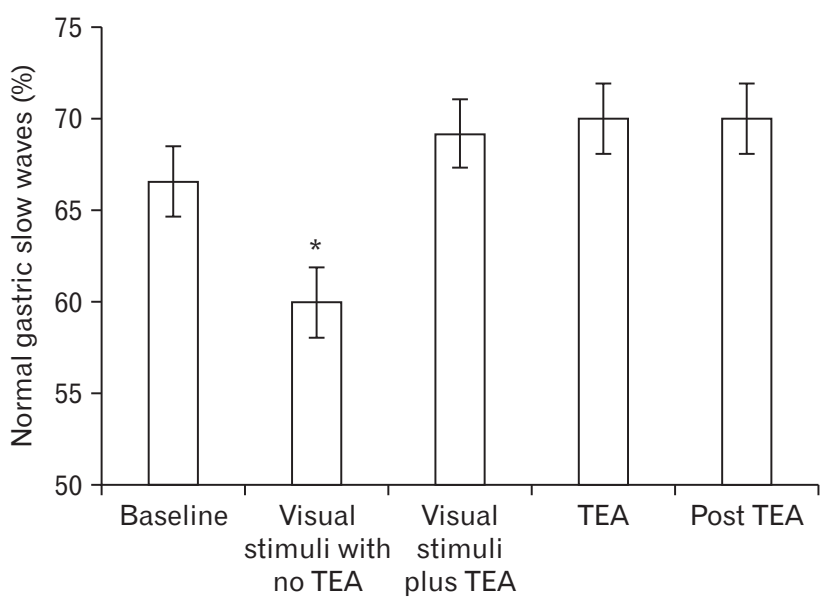

Figure 4. Percentage of normal gastric slow waves during the different phases of the experimental research protocol. ${ }^{*} P<0.05$ vs the corresponding visual stimuli with no transcutaneous electroacupuncture (TEA). ment (Fig. 5).

\section{Correlation of Frontal Asymmetries with Nausea Scores and Electrogastrography Signals}

\section{(See Fig. 6 and Table)}

Evidence of the right inferior frontal lobe activation was found at the baseline with a mean nausea score of $3.5 \pm 1.0$ and percentage of normal gastric slow waves of $66.6 \pm 4.5 \%$. After visual stimuli, a large activation of the right inferior frontal gyrus and parietal lobe were observed (AUC: $-13.89 \mathrm{nA} / \mathrm{mm}^{2}$ ) with increased nausea score of $5.9 \pm 1.5$ and decreased percentage of normal gastric slow waves of $60.0 \pm 5.7 \%$. During TEA, activation of the left interior frontal gyrus became predominant (AUC: $15.87 \mathrm{nA} / \mathrm{mm}^{2}$ ) with improved nausea scores of $3.5 \pm 1.2$ and enhanced percent-

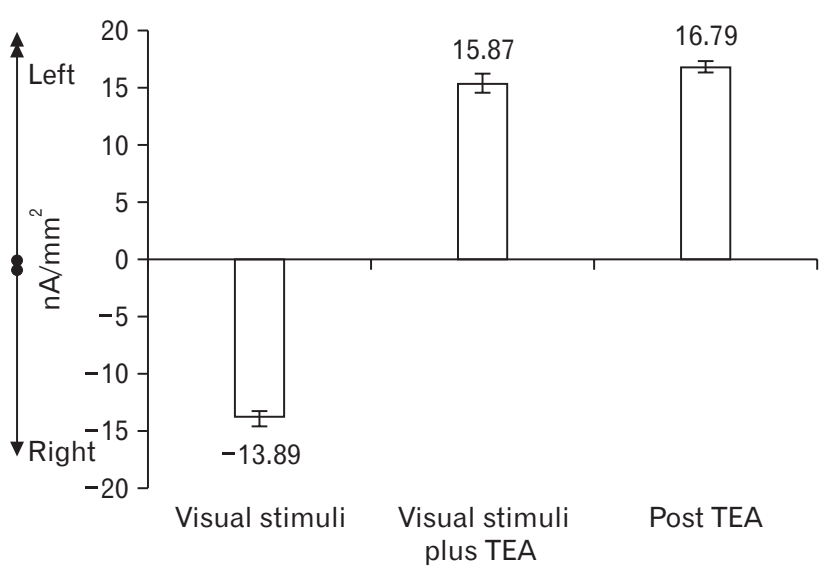

Figure 6. Electroencephalography frontal asymmetry. Cortical slow potentials of frontal lobe sources were separated between left and right regional generators, and the area under the curve for the stimulation intervals was then computed and analyzed. A negative value indicates a right dominance, a positive value a left dominance.
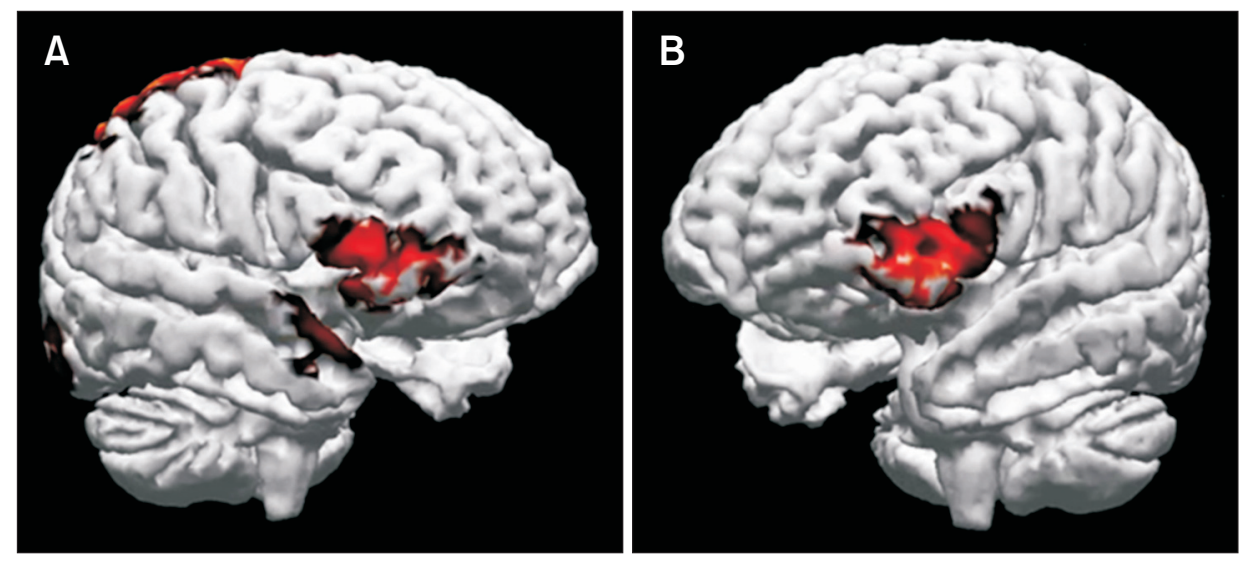

Figure 5. Neural activity source reconstruction. (A) During visual stimulation. A large activation of the right inferior frontal gyrus can be seen. Activation of parietal lobe is also evident. (B) During and post transcutaneous electroacupuncture treatment. The left inferior frontal gyrus activity was observed. 
Table. Changes in Nausea Scores and Percentage of Normal Gastric Slow Waves, and Progression of Brain Modeling During the Experimental Phases

\begin{tabular}{lccl}
\hline \multicolumn{1}{c}{ Phases } & $\begin{array}{c}\text { Nausea scores } \\
(\text { means } \pm \mathrm{SE})\end{array}$ & $\begin{array}{c}\text { Percentage of normal gastric } \\
\text { slow waves (means } \pm \mathrm{SE})\end{array}$ & Neural activity source reconstruction \\
\hline $\begin{array}{l}\text { 1. Baseline } \\
\text { 2. After visual stimuli } \\
\text { with no TEA }\end{array}$ & $5.5 \pm 1.0^{\mathrm{a}}$ & $66.6 \pm 4.5^{\mathrm{a}}$ & $\begin{array}{l}\text { Evidence of the right inferior frontal lobe activation } \\
\text { A large activation of the right inferior frontal gyrus and parietal lobe }\end{array}$ \\
$\begin{array}{l}\text { 3. During TEA } \\
\text { 4. Post TEA }\end{array}$ & $3.5 \pm 1.2^{\mathrm{a}}$ & $60.0 \pm 5.7$ & Activation of the left interior frontal gyrus \\
Continuation of the activation of the left interior frontal gyrus
\end{tabular}

${ }^{\mathrm{a} P}<0.05$ vs the corresponding visual stimuli with no transcutaneous electroacupuncture.

age of normal gastric slow waves of $69.2 \pm 4.8 \%$. After TEA was stopped, the activation of the left interior frontal gyrus was continued (AUC: $16.79 \mathrm{nA} / \mathrm{mm}^{2}$ ) with sustained amelioration of nausea scores $(2.9 \pm 1.0)$ and increased percentage of normal gastric slow waves $(69.2 \pm 4.8 \%)$. The Pearson's correlation of frontal asymmetries with nausea and the EGG signals were 0.986 and 0.993 , respectively $(P<0.05)$.

\section{Discussion}

Our study conducted on nauseated diabetic gastroparetic patients showed that: (1) visual stimuli increased nausea symptoms, impaired gastric slow waves, and activated the right inferior frontal lobe, which is presented in the literature as the area of unpleasant stimuli perception and (2) application of TEA therapy decreased the visually induced nausea symptom, prevented the impairment of gastric slow waves, and changed the dominance from right to left inferior frontal lobe activity which is related to sensing pleasant stimuli.

We found that TEA improved visual stimuli-induced nausea symptoms, which is in agreement with previously presented results showing that, acupuncture and electroacupuncture were able to improve gastric motility and alleviate symptoms of upper abdomen, including nausea and vomiting. In canines, McNearney et al, ${ }^{30}$ demonstrated that electroacupuncture at PC6 reduced vasopressininduced nausea and vomiting mediated via the vagal mechanism. Ouyang et al, ${ }^{31}$ reported that electroacupuncture at points PC6 and ST36 significantly accelerate gastric emptying in dogs via the vagal mechanism. Clinically, there is evidence that acupuncture at PC6 and ST36 improved dyspeptic symptoms including nausea and vomiting and GMA in gastroparetic patients. ${ }^{8}$ These data indicate that electroacupuncture is able to improve nausea and vomiting of different causes.

EGG is an accurate and reliable measure for studying GMA. ${ }^{12,13}$
The present study found a significant effect of the visual stimuli on GMA. The visual stimuli decreased the percentage of normal slow waves in the fasting state, attributed to an increase in bradygastria and tachygastria. The effect of stress on gastric slow waves was shown by Imai et $\mathrm{al}^{32}$ who demonstrated an increase in tachygastria with a decrease in the normal slow waves induced by optokinetic motion sickness. Also, Chen et $\mathrm{al}^{33}$ showed that audio stimulation (noise) impaired percentage of normal gastric slow wave in adolescents. In addition, Riezzo et al, ${ }^{34}$ found similar effects on gastric slow waves by different forms of stress, such as cold water and arithmetic task.

Our study demonstrated that TEA normalized visual stimuliinduced impairment of GMA. Conventional needle-based EA has been used to treat gastric dysmotility. Ouyang et $\mathrm{al}^{31}$ showed that EA at ST36 improved vagotomy-induced impairment of gastric accommodation in dogs but had no effects on gastric accommodation in normal dogs. Some other studies also found ameliorating effects of EA on GMA. EA at ST36 restored rectal distentioninduced impairment in gastric slow waves in dogs and increased the spikes in rabbits. ${ }^{35-37} \mathrm{EA}$ at ST36 improved the percentage of normal gastric slow waves as well as the percentage of tachygastria in healthy volunteers. ${ }^{38}$ Liu et al ${ }^{21}$ reported that 2-week TEA at ST36 decreased dyspepsia symptoms and elevated vagal activity in patients with functional dyspepsia. Two-week daily TEA at ST36 improved GI symptoms and quality of life in systemic sclerosis. ${ }^{22,30}$ To our knowledge, no study has ever explored the effects of TEA on visual stimuli-induced gastric slow wave abnormalities.

The cortical imaging of slow potentials in the brain as recorded by EEG showed a high correlation $(r=0.986)$ between the onset of TEA and a shift away from the right inferior frontal gyrus to the left inferior frontal gyrus. ${ }^{28,39,40}$ The left-right frontal asymmetry is most often characterized as an asymmetry in the alpha activity recorded over the F7 and F8 electrodes which are directly over the inferior frontal gyrus. Here we demonstrate that this affect ex- 
tends to nausea, to our knowledge, for the first time. This result is consistent with previous data reported by Craig. ${ }^{11}$ In our study, we explored the alpha rhythm, but found the largest effects in synchronized slow waves associated with the onset of the nausea inducing stimuli. Indeed our results were more sensitive and statistically robust than data presented based on previously conducted behavioral studies. $^{11,41}$

We understand that the lack of the Sham TEA arm of the study hampers our results. Due to the pilot nature of this project, we decided to focus on the efficacy of real TEA stimulation in order to assess whether any changes in functional brain images were able to be recorded and if there was any correlation between brain images and the different phases of the experiment. Further research should incorporate a "Sham" arm and further investigate the findings and observations we have described in this pilot study.

In summary, in patients with diabetic gastroparesis and ongoing nausea: (1) needleless TEA treatment improves nausea and gastric slow waves abnormalities exacerbated by visual stimulation. (2) TEA during visual stimulation results in the shift of dominance from right to left inferior frontal brain lobe activity. This effect is sustained post TEA and is correlated with reduced nausea and improved EGG signals.

We believe that TEA therapy is efficacious in reducing nausea in DMGP patients. These data show the importance of frontal asymmetry as a central mechanisms of nausea and how needleless transcutaneous electrical acupuncture affects this pathway. This model which we have utilized for studying nausea in DMPG could provide new insights into mechanisms and treatment targets as well as have the potential to investigate other GI related etiologies of nausea and vomiting.

Financial support: This work was supported by a seed award grant of Texas Tech University Health Sciences Center, Paul L. Foster School of Medicine (Grant No. E14033).

\section{Conflicts of interest: None.}

Author contributions: Irene Sarosiek, Gengqing Song, and Yan Sun performed the research, analyzed the nausea and EGG data, and wrote the paper; Hugo Sandoval and Stephen Sands analyzed the EEG data and helped write the EEG methodology, EEG results, and EEG discussions; Jiande Chen provided the TEA device with technical support and consults; and Richard McCallum involved in all the aspects of the study such the research study design and reviews of the paper.

\section{References}

1. Zitomer BR, Gramm HF, Kozak GP. Gastric neuropathy in diabetes mellitus: clinical and radiologic observations. Metabolism 1968;17:199211.

2. Horowitz M, O'Donovan D, Jones KL, Feinle C, Rayner CK, Samsom M. Gastric emptying in diabetes: clinical significance and treatment. Diabet Med 2002;19:177-194.

3. Horowitz M, Edelbroek M, Fraser R, Maddox A, Wishart J. Disordered gastric motor function in diabetes mellitus. Recent insights into prevalence, pathophysiology, clinical relevance, and treatment. Scand J Gastroenterol 1991;26:673-684.

4. Dundee JW, Chestnutt WN, Ghaly RG, Lynas AG. Traditional Chinese acupuncture: a potentially useful antiemetic? Br Med J (Clin Res Ed) 1986;293:583-584.

5. Ghaly RG, Fitzpatrick KT, Dundee JW. Antiemetic studies with traditional Chinese acupuncture. A comparison of manual needling with electrical stimulation and commonly used antiemetics. Anaesthesia 1987;42:1108-1110

6. Dundee JW, Ghaly RG, Fitzpatrick KT, Lynch GA, Abram WP. Acupuncture to prevent cisplatin-associated vomiting. Lancet 1987;1:1083.

7. Song GQ, Zhang N, Shi R, Chen JD. Synchronized transcutaneous electroacupuncture improves high-fat meal induced impairment of gastric slow waves and autonomic function in healthy subjects. Am J Gastroenterol 2013;108:S55.

8. Chen JD, Sarosiek I, McCallum RW, et al. Chronic electrical stimulation at acupuncture points improves dyspeptic symptoms in patients with diabetic gastroparesis. Am J Gastroenterol 2012;107:S57-S58.

9. Frøkjær JB, Egsgaard LL, Graversen C, et al. Gastrointestinal symptoms in type-1 diabetes: is it all about brain plasticity? Eur J Pain 2011;15:249257.

10. Brock C, Graversen C, Frøkjaer JB, Søfteland E, Valeriani M, Drewes AM. Peripheral and central nervous contribution to gastrointestinal symptoms in diabetic patients with autonomic neuropathy. Eur J Pain 2013;17:820-831.

11. Craig AD. How do you feel--now? The anterior insula and human awareness. Nat Rev Neurosci 2009; 10:59-70.

12. Chen JD, Schirmer BD, McCallum RW. Serosal and cutaneous recordings of gastric myoelectrical activity in patients with gastroparesis. Am J Physiol 1994;266(1 Pt 1):G90-G98.

13. Chen JD, Pan J, McCallum RW. Clinical significance of gastric myoelectrical dysrhythmias. Dig Dis 1995;13:275-290.

14. You CH, Lee KY, Chey WY, Menguy R. Electrogastrographic study of patients with unexplained nausea, bloating, and vomiting. Gastroenterology 1980;79:311-314.

15. Telander RL, Morgan KG, Kreulen DL, Schmalz PF, Kelly KA, Szurszewski JH. Human gastric atony with tachygastria and gastric retention. Gastroenterology 1978;75:497-501.

16. Geldof H, van der Schee EJ, van Blankenstein M, Grashuis JL. Electrogastrographic study of gastric myoelectrical activity in patients with unexplained nausea and vomiting. Gut 1986;27:799-808.

17. Chen J, McCallum RW. Gastric slow wave abnormalities in patients with 
gastroparesis. Am J Gastroenterol 1992;87:477-482.

18. Kiernan BD, Soykan I, Lin Z, Dale A, McCallum RW. A new nausea model in humans produces mild nausea without electrogastrogram and vasopressin changes. Neurogastroenterol Motil 1997;9:257-263.

19. Kaada B. Successful treatment of esophageal dysmotility and Raynaud's phenomenon in systemic sclerosis and achalasia by transcutaneous nerve stimulation. Increase in plasma VIP concentration. Scand J Gastroenterol 1987;22:1137-1146.

20. Maeda M, Ichiki Y, Sumi A, Mori S. A trial of acupuncture for progressive systemic sclerosis. J Dermatol 1988;15:133-140.

21. Liu S, Peng S, Hou X, Ke M, Chen JD. Transcutaneous electroacupuncture improves dyspeptic symptoms and increases high frequency heart rate variability in patients with functional dyspepsia. Neurogastroenterol Motil 2008;20:1204-1211.

22. Sallam H, McNearney TA, Doshi D, Chen JD. Transcutaneous electrical nerve stimulation (TENS) improves upper GI symptoms and balances the sympathovagal activity in scleroderma patients. Dig Dis Sci 2007;52:1329-1337.

23. Oostenveld R, Praamstra P. The five percent electrode system for high-resolution EEG and ERP measurements. Clin Neurophysiol 2001;112:713-719.

24. Michel CM, Murray MM, Lantz G, Gonzalez S, Spinelli L, Grave de Peralta R. EEG source imaging. Clin Neurophysiol 2004;115:2195-222.

25. Hennighausen E, Heil M, Rösler F. A correction method for DC drift artifacts. Electroencephalogr Clin Neurophysiol 1993;86:199-204.

26. Fonov V, Evans AC, Botteron K, et al. Unbiased average age-appropriate atlases for pediatric studies. Neuroimage 2011;54:313-327.

27. Fuchs M, Kastner J, Wagner M, Hawes S, Ebersole JS. A standardized boundary element method volume conductor model. Clin Neurophysiol 2002;113:702-712.

28. Pascual-Marqui RD. Standardized low-resolution brain electromagnetic tomography (sLORETA): technical details. Methods Find Exp Clin Pharmacol 2002;24(suppl D):5-12.

29. Wewers ME, Lowe NK. A critical review of visual analogue scales in the measurement of clinical phenomena. Res Nurs Health 1990;13:227-236.

30. McNearney TA, Sallam HS, Hunnicutt SE, Doshi D, Chen JD. Prolonged treatment with transcutaneous electrical nerve stimulation (TENS) modulates neuro-gastric motility and plasma levels of vasoactive intestinal peptide (VIP), motilin and interleukin-6 (IL-6) in systemic sclerosis. Clin Exp Rheumatol 2013;31(2 suppl 76):140-150.

31. Ouyang H, Xing J, Chen J. Electroacupuncture restores impaired gastric accommodation in vagotomized dogs. Dig Dis Sci 2004;49:1418-1424.

32. Imai K, Kitakoji H, Sakita M. Gastric arrhythmia and nausea of motion sickness induced in healthy Japanese subjects viewing an optokinetic rotating drum. J Physiol Sci 2006;56:341-345.

33. Chen DD, Xu X, Zhao Q, Yin J, Sallam H, Chen JD. Effects of audio stimulation on gastric myoelectrical activity and sympathovagal balance in healthy adolescents and adults. J Gastroenterol Hepatol 2008;23:141149.

34. Riezzo G, Porcelli P, Guerra V, Giorgio I. Effects of different psychophysiological stressors on the cutaneous electrogastrogram in healthy subjects. Arch Physiol Biochem 1996;104:282-286.

35. Niu WX, He GD, Liu H, Qin XY. Effects and probable mechanisms of electroacupuncture at the Zusanli point on upper gastrointestinal motility in rabbits. J Gastroenterol Hepatol 2007;22:1683-1689.

36. Chen J, Song GQ, Yin J, Koothan T, Chen JD. Electroacupuncture improves impaired gastric motility and slow waves induced by rectal distension in dogs. Am J Physiol Gastrointest Liver Physiol 2008;295:G614G620.

37. Yin J, Chen J, Chen JD. Ameliorating effects and mechanisms of electroacupuncture on gastric dysrhythmia, delayed emptying, and impaired accommodation in diabetic rats. Am J Physiol Gastrointest Liver Physiol 2010;298:G563-G570.

38. Chang CS, Chou JW, Ko CW, Wu CY, Chen JD. Cutaneous electrical stimulation of acupuncture points may enhance gastric myoelectrical regularity. Digestion 2002;66:106-111.

39. Allen JJ, Kline JP. Frontal EEG asymmetry, emotion, and psychopathology: the first, and the next 25 years. Biol Psychol 2004;67:1-5.

40. Harmon-Jones E, Gable PA, Peterson CK. The role of asymmetric frontal cortical activity in emotion-related phenomena: a review and update. Biol Psychol 2010;84:451-462.

41. Al Omran Y, Aziz Q. Functional brain imaging in gastroenterology: to new beginnings. Nat Rev Gastroenterol Hepatol 2014;11:565-576. 